\title{
Ultra-adherence with Electronic Pills-box may Reveal Poor Adherence
}

\author{
Marie Paule Schneider ${ }^{1}$, Matthias Cavassini ${ }^{2}$, Olivier Bugnon ${ }^{1,3}$ and Nicolas Senn ${ }^{4 *}$ \\ ${ }^{1}$ Community Pharmacy, Department of Ambulatory Care \& Community Medicine, University of Lausanne, Switzerland \\ ${ }^{2}$ Service of Infectious Disease, Centre Hospitalier Universitaire Vaudois and University of Lausanne, Switzerland \\ ${ }^{3}$ Community Pharmacy Research Unit, Pharmaceutical Sciences Section, Universities of Geneva \& Lausanne, Switzerland \\ ${ }^{4}$ Department of Ambulatory Care, University of Lausanne, Switzerland
}

\begin{abstract}
One of the challenges of antiretroviral treatments (CART) in HIV is to achieve optimal and persistent adherence. In order to achieve this, electronic pill-boxes used in conjunction with motivational interviewing have shown promising results in monitoring and improving patient's adherence to treatments. This study reports a case where the patient was opening every day on time his pill-box to discard systematically the tablets in order to hide consciously his nonadherence. A too regular pattern in drug intake according to the electronic pill-box should alert health professionals for the potential risk of distorted adherence results.
\end{abstract}

\section{Introduction}

One of the challenges of chronic therapies is to achieve optimal and persistent adherence. This is especially true for combined antiretroviral treatments (cART) in HIV [1]. Several collaborative strategies have been developed between pharmacists and physicians in order to improve adherence and continuity of care. Among them, electronic pill-boxes used in conjunction with motivational interviewing have shown promising results in monitoring and improving patient's adherence to treatments [2]. Thanks to such a technology, it becomes possible to give patients longitudinal feedback on the way they manage their treatment to reinforce their adherent behavior, or understand their issues in case of nonadherence in order to find individual adjustments [3]. The electronic monitor MEMS ${ }^{\mathrm{Tw}}$ (Medication Event Monitoring System, AARDEX Group, MWV Healthcare) has been used with success in routine clinical care. Other devices as Wisepill or POEMS are promising too but need to be further studied [4-6].

\section{Case Report}

A 46-year old single man was diagnosed with HIV (grade C III) in 1999. Although the patient had difficulties accepting the diagnosis and suffered from depression, cART (zidovudine/lamivudine, nelfinavir) was initiated in 1999 because of an extremely weak immune system (CD4 count of 28 cells/ mm3). The patient refused psychiatric followup. Poor adherence was suspected in 2002 as viral load increased from $40^{\prime} 000$ to $322^{\prime} 000$ copies $/ \mathrm{ml}$ and CD4 count dropped down progressively from $245 \mathrm{cell} / \mathrm{mm}^{3}$ (14\%) to $132 \mathrm{cell} / \mathrm{mm}^{3}$ (8\%) over 1 year. Plasma nelfinavir drug levels were repeatedly very low and no resistance mutations were identified. Furthermore, the patient never reported any side effects of the treatment. He was referred to the on-site medication adherence clinic with the aim of supporting his adherence instead of escalating to a new cART.

On the first medication adherence visit with the pharmacist, communication was poor. The patient was restless, pale, distressed and totally unwilling to talk about his cART. The pharmacist used motivational interviewing skills for establishing the link with the patient and for decreasing mistrust. The only gathered information was: a) the patient was in conflict with his physician as he felt confronted by the non-adherence suspicion; and b) the patient denied his HIV-status. The patient agreed on using electronic pill-boxes as he wanted to prove that he was taking his cART exactly as prescribed. Three weeks later, he came back to the second scheduled adherence visit with a similar defensive behavior but he engaged in a short discussion. One month later, at his third appointment, the patient was still in the same stage. Surprisingly, during these 3.5 months, the patient showed a perfect cART adherence according to the pill-boxes records. Electronic monitors had been systematically opened at $8.30 \mathrm{am}$ and $8.30 \mathrm{pm}$ except on two occasions (Figure 1a). By way of comparison, Figure 1b shows a standard BID cART intake pattern. Afterwards, the patient decided to exit the adherence clinic prematurely but agreed on a close follow-up with his physician. At the next clinic attendance, for the very first time, he confessed his nonadherence and the fact that he was systematically discarding the tablets after opening the pill-box.

\section{Discussion}

This case report is of importance, not because it describes a patient who does not take his medication, which is often the case, but because the pattern observed in the pill box records is so specific (compared to a standard pattern, as shown in Figure 1b) that it allows by itself to suspect nonadherence despite daily box openings. To our knowledge, this was never described in the literature. This adds value to the interpretation of electronic pill box. In sum, a too regular pattern of drug intake according to the electronic pill-box records in conjunction with patient's distressed behavior during interviews should alert health professionals for the potential risk of distorted adherence results. This case also shows that healthcare givers can work in seamless care and support patient's confidence in addressing a sensitive but detrimental behavior using motivational interviewing skills. Indeed, the patient never broke up his relationship to the physician and pharmacist despite the conflict. According to the literature, the major perceived drawback of electronic monitoring is that it does not prove drug ingestion, mainly because of inconsistent use, intentional mishandling or even fraud $[7,8]$. However, we believe that this limitation could be mainly overcome

*Corresponding author: Nicolas Senn, Department of Ambulatory Care And Community Medicine, University of Lausanne, Switzerland, Tél: +41213140406: E-mail: nicolas.senn@hospvd.ch

Received July 01, 2013; Accepted October 17, 2013; Published October 24 2013

Citation: Schneider MP, Cavassini M, Bugnon O, Senn N (2013) Ultra-adherence with Electronic Pills-box may Reveal Poor Adherence. J Gen Pract 1: 127. doi: 10.4172/2329-9126.1000127

Copyright: @ 2013 Schneider MP, et al. This is an open-access article distributed under the terms of the Creative Commons Attribution License, which permits unrestricted use, distribution, and reproduction in any medium, provided the original author and source are credited. 
Citation: Schneider MP, Cavassini M, Bugnon O, Senn N (2013) Ultra-adherence with Electronic Pills-box may Reveal Poor Adherence. J Gen Pract 1: 127 . doi: $10.4172 / 2329-9126.1000127$

Page 2 of 2

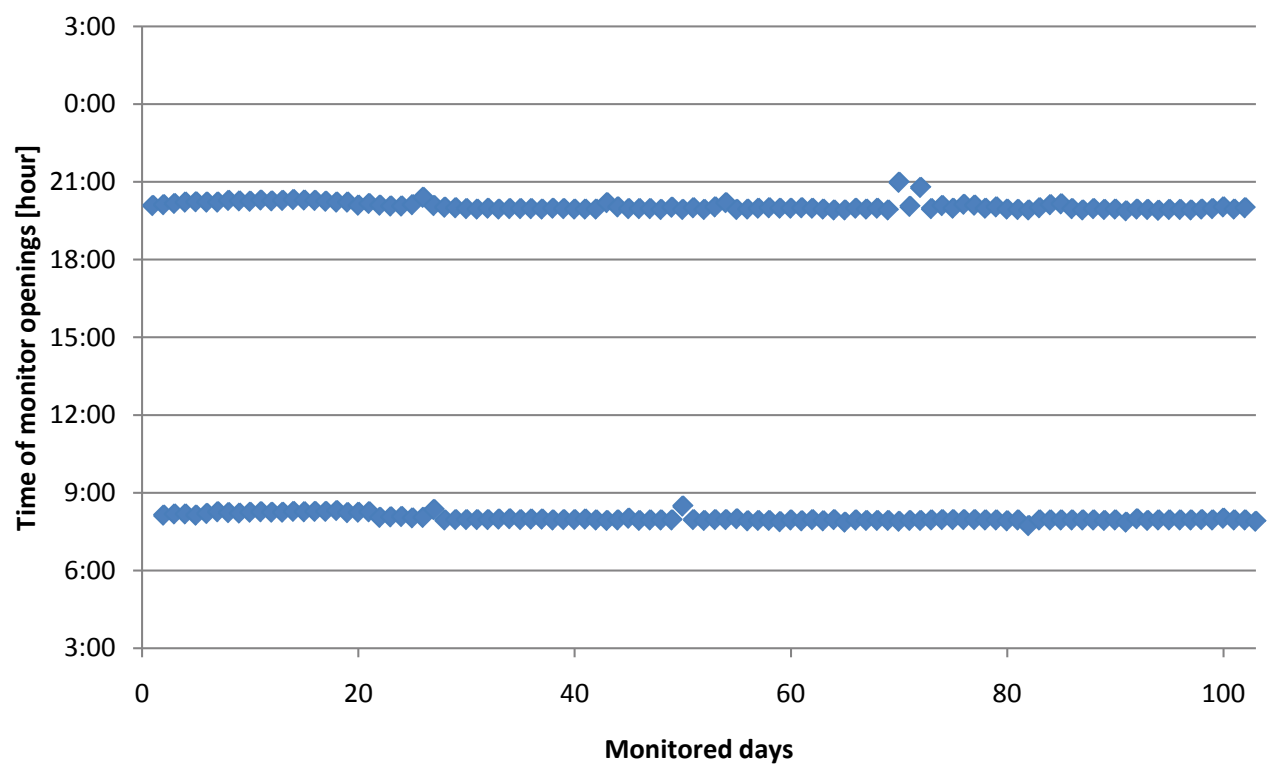

Figure 1a: Records of electronic pill-boxes over a 100-day monitored period; each dot represents the day and the time of an opening. A: Our case showing an extremely regular pattern of dose intake (no missed dose).

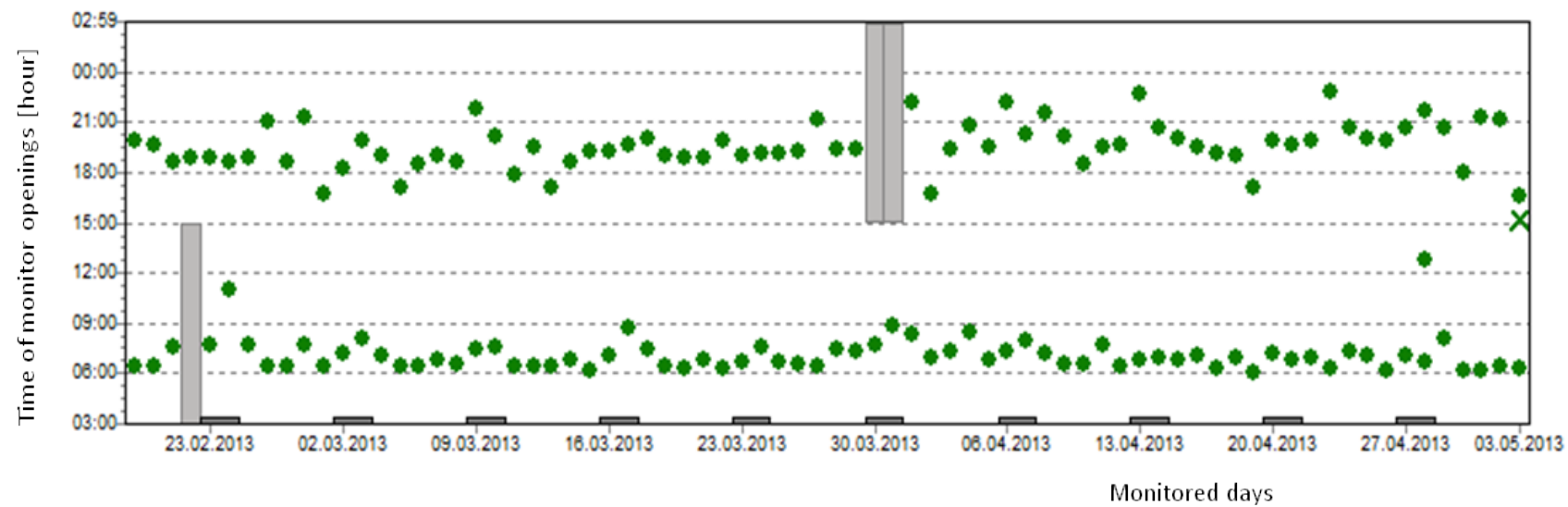

Figure 1b: A representative example of a common BID cART intake pattern, with most doses taken within an interval of \pm 3 hours (grey bars represent missed doses).

through a structured, empathic, patient-centered, sound adherence interview as shown in this case associated to a careful interpretation of pill box records. Additionally, the use of Therapeutic Drug Monitoring (TDM) in conjunction with electronic pill-monitoring might improve detection of suboptimal adherence levels.

\section{Acknowlegment}

Nicolas Senn is supported by an academic "bridge-relève" scholarship provided by the Leenaards Foundation (Lausanne, Switzerland).

\section{References}

1. Glass TR, de Geest S, Hirschel B, Battegay M, Furrer H, et al. (2008) Selfreported non-adherence to antiretroviral therapy repeatedly assessed by two questions predicts treatment failure in virologically suppressed patients. Antivir Ther 13: 77-85

2. Krummenacher I, Cavassini M, Bugnon O, Schneider MP (2011) An interdisciplinary HIV-adherence program combining motivational interviewing and electronic antiretroviral drug monitoring. AIDS Care 23: 550-561.

3. Demonceau J, Ruppar T, Kristanto P, Hughes DA, Fargher E, et al. (2013) Identification and assessment of adherence-enhancing interventions in studies assessing medication adherence through electronically compiled drug dosing histories: a systematic literature review and meta-analysis. Drugs 73: 545-562.

4. Haberer JE, Kahane J, Kigozi I, Emenyonu N, Hunt P, et al. (2010) Real-time adherence monitoring for HIV antiretroviral therapy. AIDS Behav 14: 1340-1346.

5. Arnet I, Walter PN, Hersberger KE (2013) Polymedication Electronic Monitoring System (POEMS) - a new technology for measuring adherence. Front Pharmacol 4: 26.

6. Lehmann A, Aslani P, Ahmed R, Celio J, Gauchet A, et al. (2013) Assessing Medication Adherence: Options to Consider. Int J Clin Pharmacol.

7. Bova CA, Fennie KP, Knafl GJ, Dieckhaus KD, Watrous E, et al. (2005) Use of electronic monitoring devices to measure antiretroviral adherence: practical considerations. AIDS Behav 9: 103-110.

8. Denhaerynck K, Schäfer-Keller P, Young J, Steiger J, Bock A, et al. (2008) Examining assumptions regarding valid electronic monitoring of medication therapy: development of a validation framework and its application on a European sample of kidney transplant patients. BMC Med Res Methodol 8: 5. 